\title{
Association between Serum Testosterone Levels and Body Composition among Men 20-59 Years of Age
}

\author{
Jiajie Ye, ${ }^{1}$ Xiaojun Zhai, ${ }^{2}$ Jinxiao Yang, ${ }^{3}$ and Zhongxin Zhu $\mathbb{D}^{4,5}$ \\ ${ }^{1}$ Liaoning University of Traditional Chinese Medicine, Shenyang, Liaoning 110847, China \\ ${ }^{2}$ Department of Orthopedics, The Affiliated Jiangnan Hospital of Zhejiang Chinese Medical University, Hangzhou, \\ Zhejiang 311200, China \\ ${ }^{3}$ Department of Urology, The Xiaoshan Affiliated Hospital of Wenzhou Medical University, Hangzhou, Zhejiang 311200, China \\ ${ }^{4}$ Department of Osteoporosis Care and Control, The Xiaoshan Affiliated Hospital of Wenzhou Medical University, Hangzhou, \\ Zhejiang 311200, China \\ ${ }^{5}$ Clinical Research Center, The Xiaoshan Affiliated Hospital of Wenzhou Medical University, Hangzhou, Zhejiang 311200, China
}

Correspondence should be addressed to Zhongxin Zhu; orthozzx@163.com

Received 2 May 2021; Revised 26 August 2021; Accepted 13 September 2021; Published 20 September 2021

Academic Editor: Daniela Merlotti

Copyright ( 2021 Jiajie Ye et al. This is an open access article distributed under the Creative Commons Attribution License, which permits unrestricted use, distribution, and reproduction in any medium, provided the original work is properly cited.

\begin{abstract}
Introduction. Sex hormones play an important role in the development and maintenance of bone and muscle mass. However, studies regarding serum testosterone levels, osteoporosis, and sarcopenia in men are relatively sparse and have led to contradictory conclusions. Therefore, this study aimed to investigate the association between serum testosterone levels and body composition, including bone mineral density (BMD), appendicular lean mass index (ALMI), and appendicular fat mass index (AFMI), among men 20-59 years of age through a cross-sectional analysis of the National Health and Nutrition Examination Survey. Materials and Methods. Our analysis was based on the data for 3,875 men, 20-59 years of age. Weighted multiple regression analyses were used to estimate the independent association between serum testosterone levels and body composition. Weighted generalized additive models and smooth curve fittings were used to characterize the nonlinear associations between them. Results. The association between the serum testosterone level and lumbar BMD was positive in each multivariable linear regression model. In the model adjusted for age and race, the serum testosterone level was negatively associated with ALMI. However, in the models adjusted for body mass index, this association became positive. In addition, the association between the serum testosterone level and AFMI was negative in each multivariable linear regression model. Conclusion. Our study demonstrated a positive association of serum testosterone level with lumbar BMD and ALMI, and a negative association with AFMI, among men 20-59 years of age, suggesting that increasing testosterone levels may be beneficial to skeletal health in young and middle-aged men with low testosterone levels.
\end{abstract}

\section{Introduction}

With aging of the general population, the prevalence of sarcopenia and osteoporosis is likely to increase substantially over the coming decades [1]. Sarcopenia is defined as the loss of skeletal muscle mass, quality, and strength, whereas osteoporosis is characterized by low bone mass and deterioration of the microarchitecture of bone [2, 3]. Both conditions are associated with devastating consequences, such as an increased risk of frailty, fragility fracture, multimorbidity, and other adverse outcomes [4]. Therefore, understanding the risk factors for these two conditions is essential for their prevention, early diagnosis, and management.

Sex hormones play an important role in the development and maintenance of bone and muscle mass $[5,6]$. As one of the representative sex hormones, testosterone has recently been shown to be involved in several metabolic functions in males [7]. Adult hypogonadism is a well-known risk factor in the development of secondary osteoporosis [8]. While menopause-related estrogen deficiency is a well-studied risk factor for osteoporosis in women, data regarding serum 
testosterone levels and osteoporosis in men are less well known, especially in younger men $<60$ years of age. Similarly, studies investigating the efficacy of testosterone on muscle mass and function have yielded contradictory conclusions based on the method of testing and study cohort [9]. What is known is that lean mass and bone mass reach their peak in young adulthood in men followed by a slow rate decrease from middle-age onward $[10,11]$. Therefore, the balance between the gain and loss of lean mass and bone mass during these periods may play a crucial role in the onset of sarcopenia and osteoporosis at an older age. Accordingly, this study aimed to evaluate the association between the serum testosterone level and body composition, including lumbar bone mineral density (BMD), appendicular lean mass index (ALMI), and appendicular fat mass index (AFMI) in younger individuals rather than elderly men, through a cross-sectional analysis of data from the National Health and Nutrition Examination Survey (NHANES).

\section{Materials and Methods}

2.1. Study Population. NHANES surveys have been conducted every 2 years in a nationally representative sample of the noninstitutionalized US population since 1999. To serve as a population-level assessment, the NHANES uses a complex, stratified, multistage probability sampling design. Data used in our study were obtained from the three waves of the NHANES conducted between 2011 and 2016.

The population was limited to men, $20-59$ years of age $(n=5,540)$. Individuals with missing serum testosterone level data $(n=556)$, lumbar BMD data $(n=573)$, appendicular lean or fat data $(n=418)$, body mass index (BMI), or height data $(n=11)$, as well as patients with cancer $(n=94)$, were excluded. We further excluded 13 participants who had ever been prescribed medicine for osteoporosis or had ever taken prednisone or cortisone daily. After screening, the data for 3,875 men were included in the final analysis. All protocols of the NHANES were approved by the National Center for Health Statistics (NCHS), and all participants provided written consent for the use of their data for research.

2.2. Study Variables. The exposure variable in this study was the serum testosterone level. Serum samples were firstly collected between 8.30 a.m. and 11.30 a.m. following an overnight fast. Based on the reference method of the $\mathrm{Na}$ tional Institute for Standards and Technology, the concentration of serum testosterone was measured using the isotope dilution-liquid chromatography tandem mass spectrometry (ID-LC-MS/MS) method, which was optimized for higher sample throughput [12]. Liquid-liquid extraction of serum was employed to isolate the steroid. As an internal standard, stable isotope-labeled testosterone was used to correct for sample recovery during the sample preparation process.

The outcome variable was body composition, including lumbar BMD, ALMI, and AFMI. Body composition was measured by dual-energy X-ray absorptiometry whole-body scans using Hologic Discovery model A densitometers (Hologic, Inc., Bedford, MA, USA) and analyzed using APEX software (version 4.0; Hologic, Inc., Bedford, MA, USA). In this study, ALMI was calculated as the appendicular lean mass $(\mathrm{kg})$ divided by height squared $\left(\mathrm{m}^{2}\right)$ and AFMI as the appendicular fat mass ( $\mathrm{kg}$ ) divided by height squared $\left(\mathrm{m}^{2}\right)$.

Demographic variables including age, race/ethnicity, education level, income-to-poverty ratio, smoking status, drinking behavior, status of moderate activities, and calcium supplement use during the 30-day period prior to the survey date were obtained from the self-reported questionnaire. Body mass index (BMI) data were collected in the mobile examination center. As part of the standard biochemistry profile, blood urea nitrogen, serum uric acid, total protein, serum phosphorus, and serum calcium are included. Moreover, the serum estradiol level was also included. Details of serum testosterone levels, body composition measurements, and other covariate acquisition processes are available at https://www.cdc.gov/nchs/nhanes/.

2.3. Statistical Analyses. All estimates were calculated accounting for the NHANES sample weights, following the guidelines edited by the NCHS [13]. Weighted multiple regression analyses were used to estimate the independent association between serum testosterone levels and body composition. Three models were used to provide statistical inference: model 1, adjusted for age and race; model 2, adjusted for age, race, and BMI; and model 3 , adjusted for all covariates.

To address potential nonlinearities, weighted generalized additive models and smooth curve fitting were performed. Statistical analyses were performed using Empower software (https://www.empowerstats.com; X\&Y solutions, Inc., Boston, MA) and R version 3.4.3 (https://www.R-project. org, The R Foundation), with a $P$ value $<0.05$ as statistically significant.

\section{Results}

A total of 3,875 participants (20-59 years of age) were included in our analysis, with the weighted characteristics of the participants subclassified based on serum testosterone levels quartiles (Q1: $\leq 300.0 \mathrm{ng} / \mathrm{dL}$; Q2: $>300.0, \leq 398.8 \mathrm{ng} / \mathrm{dL}$; Q3: >399.0, $\leq 521.0 \mathrm{ng} / \mathrm{dL}$; and Q4: >521.0 $\mathrm{ng} / \mathrm{dL}$ ), as shown in Table 1. There were significant differences in baseline characteristics between participants in the different serum testosterone levels quartiles, with the exception of race, education level, moderate activities, and calcium supplement use. Compared with the Q1 group, participants in the other quartile groups were younger, had increased total protein levels and lumbar BMD, and had decreased BMI, serum uric acid levels, AFMI, and ALMI.

3.1. Association between Serum Testosterone Level and Lumbar BMD. The association between the serum testosterone level and lumbar BMD was positive for each 
TABLE 1: Weighted characteristics of study population based on serum testosterone levels quartiles.

\begin{tabular}{|c|c|c|c|c|c|}
\hline Serum testosterone levels (ng/dL) & Q1 $(\leq 300.0)$ & Q2 $(>300, \leq 398.8)$ & Q3 $(>399.0, \leq 521)$ & $\mathrm{Q} 4(>521.0)$ & $P$ value \\
\hline Age (years) & $40.8 \pm 10.8$ & $39.6 \pm 11.5$ & $38.2 \pm 11.7$ & $36.2 \pm 11.9$ & $<0.001$ \\
\hline Race/ethnicity (\%) & & & & & 0.2914 \\
\hline Non-Hispanic white & 62.6 & 60.5 & 63.2 & 60.0 & \\
\hline Non-Hispanic black & 9.2 & 10.2 & 9.5 & 13.0 & \\
\hline Mexican American & 11.6 & 12.6 & 11.2 & 11.1 & \\
\hline Other race/ethnicity & 16.5 & 16.6 & 16.1 & 15.9 & \\
\hline Education level (\%) & & & & & 0.4021 \\
\hline Less than high school & 14.8 & 14.7 & 16.1 & 16.5 & \\
\hline High school & 22.0 & 24.1 & 22.0 & 24.9 & \\
\hline More than high school & 63.2 & 61.1 & 61.9 & 58.6 & \\
\hline Body mass index $\left(\mathrm{kg} / \mathrm{m}^{2}\right)$ & $31.9 \pm 6.7$ & $29.2 \pm 5.2$ & $27.3 \pm 4.6$ & $25.7 \pm 4.6$ & $<0.001$ \\
\hline Income-to-poverty ratio & $3.1 \pm 1.6$ & $3.0 \pm 1.6$ & $2.9 \pm 1.6$ & $2.7 \pm 1.6$ & $<0.001$ \\
\hline Moderate activities (\%) & & & & & 0.399 \\
\hline Both moderate recreational and work activities & 21.6 & 22.2 & 22.9 & 24.2 & \\
\hline Moderate recreational activities & 23.6 & 22.9 & 24.8 & 24.2 & \\
\hline Moderate work activities & 25.6 & 26.0 & 21.3 & 23.0 & \\
\hline No moderate recreational or work activities & 29.2 & 28.9 & 30.9 & 28.6 & \\
\hline Smoking status $(\%)$ & & & & & $<0.001$ \\
\hline Everyday & 12.4 & 17.0 & 18.2 & 22.5 & \\
\hline Some day & 4.9 & 5.6 & 6.0 & 9.1 & \\
\hline Past smoking & 27.0 & 20.8 & 21.7 & 19.1 & \\
\hline Nonsmoker or smoked less than 100 cigarettes in life & 55.7 & 56.6 & 54.0 & 49.3 & \\
\hline Had $\geq 12$ alcohol drinks per year $(\%)$ & & & & & 0.026 \\
\hline Yes & 80.69 & 82.88 & 83.41 & 85.54 & \\
\hline No & 15.51 & 12.24 & 12.60 & 10.10 & \\
\hline Not recorded & 3.80 & 4.88 & 3.99 & 4.36 & \\
\hline Blood urea nitrogen $(\mathrm{mmol} / \mathrm{L})$ & $4.82 \pm 1.64$ & $4.87 \pm 1.48$ & $4.80 \pm 1.39$ & $4.67 \pm 1.48$ & 0.029 \\
\hline Serum uric acid $(\mu \mathrm{mol} / \mathrm{L})$ & $378.3 \pm 78.2$ & $364.8 \pm 69.0$ & $355.9 \pm 63.7$ & $335.7 \pm 64.6$ & $<0.001$ \\
\hline Total protein $(\mathrm{g} / \mathrm{L})$ & $71.6 \pm 4.5$ & $71.7 \pm 4.5$ & $71.8 \pm 4.3$ & $72.3 \pm 4.4$ & 0.006 \\
\hline Serum estradiol level (pg/mL) & $21.2 \pm 8.4$ & $22.4 \pm 7.2$ & $24.1 \pm 7.3$ & $29.7 \pm 10.1$ & $<0.001$ \\
\hline Serum phosphorus $(\mathrm{mmol} / \mathrm{L})$ & $1.21 \pm 0.18$ & $1.21 \pm 0.18$ & $1.19 \pm 0.18$ & $1.19 \pm 0.19$ & 0.042 \\
\hline Serum calcium $(\mathrm{mmol} / \mathrm{L})$ & $2.36 \pm 0.09$ & $2.36 \pm 0.08$ & $2.36 \pm 0.08$ & $2.37 \pm 0.08$ & $<0.001$ \\
\hline Calcium supplement use 30 days (\%) & & & & & 0.2892 \\
\hline$\leq 400(\mathrm{mg})$ & 24.6 & 25.0 & 24.8 & 23.1 & \\
\hline$>400(\mathrm{mg})$ & 12.1 & 10.3 & 10.0 & 9.0 & \\
\hline Not recorded & 63.3 & 64.7 & 65.2 & 67.9 & \\
\hline Appendicular fat mass index $\left(\mathrm{kg} / \mathrm{m}^{2}\right)$ & $4.4 \pm 1.8$ & $3.7 \pm 1.2$ & $3.3 \pm 1.2$ & $2.9 \pm 1.1$ & $<0.001$ \\
\hline Appendicular lean mass index $\left(\mathrm{kg} / \mathrm{m}^{2}\right)$ & $9.4 \pm 1.5$ & $9.0 \pm 1.3$ & $8.7 \pm 1.2$ & $8.5 \pm 1.3$ & $<0.001$ \\
\hline Lumbar bone mineral density $\left(\mathrm{mg} / \mathrm{cm}^{2}\right)$ & $1017.3 \pm 154.6$ & $1017.5 \pm 153.4$ & $1035.3 \pm 149.3$ & $1047.6 \pm 149.5$ & $<0.001$ \\
\hline
\end{tabular}

Mean \pm SD for continuous variables: $P$ value was calculated by the weighted linear regression model. $\%$ for categorical variables: $P$ value was calculated by the weighted chi-square test.

multivariable linear regression model (Table 2). Moreover, the trend remained significant among the different serum testosterone level quartile groups ( $P$ for trend $<0.001$ ). In the subgroup analysis stratified by age and race, this positive association remained in the 20- to 39-year group $(\beta=0.07$, $95 \%$ CI: 0.02 to 0.11$)$, and the non-Hispanic black $(\beta=0.09$, 95\% CI: 0.01 to 0.17$)$ group, but not in the 40 - to 59 -year group ( $\beta=0.01,95 \% \mathrm{CI}$ : -0.04 to 0.05$)$.

3.2. Association between Serum Testosterone Level and ALMI. In the model adjusted for age and race (Table 3 ), the serum testosterone level was negatively associated with ALMI $(\beta=-0.0022,95 \% \mathrm{CI}:-0.0024$ to -0.0019$)$. However, in the models adjusted for BMI, this association became positive (model 2: $\beta=0.0005,95 \%$ CI: 0.0004 to 0.0006 ; model 3: $\beta=0.0007,95 \% \mathrm{CI}: 0.0005$ to 0.0008$)$. The $P$ for trend remained significant in each model. In the subgroup analysis stratified by age and race, this positive association remained in both the $20-39$ years $(\beta=0.0007,95 \% \mathrm{CI}$ : 0.0005 to $0.0009)$ and $40-59$ years $(\beta=0.0006,95 \% \mathrm{CI}: 0.0004$ to $0.0009)$ groups and in the non-Hispanic white $(\beta=0.0009$, 95\% CI: 0.0007 to 0.0012 ) and non-Hispanic black ( $\beta=0.0004,95 \% \mathrm{CI}: 0.0001$ to 0.0007$)$ groups in the fully adjusted model.

3.3. Association between Serum Testosterone Level and AFMI. The association between the serum testosterone level and AFMI was negative in each multivariable linear regression model (Table 4 ). The $P$ for trend remained significant in each model. In the subgroup analysis stratified by age and race, this negative association remained in both the 20-39 years $(\beta=-0.0004,95 \% \mathrm{CI}:-0.0006$ to -0.0002$)$ and $40-59$ years 
TABLe 2: Association between serum testosterone levels (ng/dL) and lumbar bone mineral density $\left(\mathrm{mg} / \mathrm{cm}^{2}\right)$.

\begin{tabular}{|c|c|c|c|}
\hline & $\begin{array}{c}\text { Model 1 } \\
\beta(95 \% \text { CI })\end{array}$ & $\begin{array}{c}\text { Model } 2 \\
\beta(95 \% \text { CI })\end{array}$ & $\begin{array}{c}\text { Model 3 } \\
\beta(95 \% \text { CI })\end{array}$ \\
\hline Serum testosterone levels (ng/dL) & $0.04(0.02,0.07)^{* *}$ & $0.07(0.04,0.10)^{* * *}$ & $0.04(0.01,0.07)^{* *}$ \\
\hline $\begin{array}{l}\text { Serum testosterone levels (quartile) } \\
\text { Q1 }(\leq 300.0) \\
\text { Q2 }(>300, \leq 398.8) \\
\text { Q3 }(>399.0, \leq 521) \\
\text { Q4 }(>521.0) \\
P \text { for trend }\end{array}$ & $\begin{array}{c}\text { Reference } \\
-0.93(-14.11,12.25) \\
16.20(3.03,29.37) \\
24.27(10.93,37.62) \\
<0.001\end{array}$ & $\begin{array}{c}\text { Reference } \\
3.83(-9.54,17.20) \\
24.14(10.39,37.88) \\
34.84(20.49,49.18) \\
<0.001 \\
\end{array}$ & $\begin{array}{c}\text { Reference } \\
0.20(-13.24,13.63) \\
19.28(5.30,33.26) \\
23.59(8.10,39.08) \\
<0.001 \\
\end{array}$ \\
\hline $\begin{array}{l}\text { Stratified by age } \\
20-39 \text { years } \\
40-59 \text { years }\end{array}$ & $\begin{array}{c}0.06(0.03,0.10)^{* *} \\
0.02(-0.03,0.06)\end{array}$ & $\begin{array}{c}0.08(0.05,0.12)^{* * *} \\
0.04(-0.00,0.09)\end{array}$ & $\begin{array}{c}0.07(0.02,0.11)^{* *} \\
0.01(-0.04,0.05)\end{array}$ \\
\hline $\begin{array}{l}\text { Stratified by race } \\
\text { Non-Hispanic white } \\
\text { Non-Hispanic black } \\
\text { Mexican American } \\
\text { Other race/ethnicity }\end{array}$ & $\begin{array}{l}0.05(0.00,0.09)^{*} \\
0.05(-0.02,0.11) \\
0.04(-0.02,0.10) \\
0.04(-0.01,0.09)\end{array}$ & $\begin{array}{c}0.06(0.01,0.11)^{*} \\
0.09(0.02,0.16)^{* *} \\
0.05(-0.02,0.11) \\
0.07(0.02,0.12)^{*}\end{array}$ & $\begin{array}{l}0.04(-0.02,0.09) \\
0.09(0.01,0.17)^{*} \\
0.02(-0.05,0.09) \\
0.03(-0.03,0.09)\end{array}$ \\
\hline
\end{tabular}

Model 1: age and race were adjusted. Model 2: age, race, and body mass index were adjusted. Model 3: age, race, body mass index, education level, income-topoverty ratio, smoking status, drinking behavior, moderate activities, calcium supplement use, blood urea nitrogen, serum uric acid, total protein, serum estradiol level, serum phosphorus, and serum calcium were adjusted. In the subgroup analysis stratified by race, the model is not adjusted for the stratification variable itself. ${ }^{*} P<0.05,{ }^{* *} P<0.01$, and ${ }^{* * *} P<0.001$.

TAвle 3: Association between serum testosterone levels $(\mathrm{ng} / \mathrm{dL})$ and appendicular lean mass index $\left(\mathrm{kg} / \mathrm{m}^{2}\right)$.

\begin{tabular}{|c|c|c|c|}
\hline & $\begin{array}{c}\text { Model } 1 \\
\beta(95 \% \mathrm{CI})\end{array}$ & $\begin{array}{c}\text { Model } 2 \\
\beta(95 \% \text { CI }) \\
\end{array}$ & $\begin{array}{c}\text { Model } 3 \\
\beta(95 \% \text { CI }) \\
\end{array}$ \\
\hline Serum testosterone levels (ng/dL) & $-0.0022(-0.0024,-0.0019)^{* * *}$ & $0.0005(0.0004,0.0006)^{* * *}$ & $0.0007(0.0005,0.0008)^{* * *}$ \\
\hline $\begin{array}{l}\text { Serum testosterone levels (quartile) } \\
\text { Q1 }(\leq 300.0) \\
\text { Q2 }(>300, \leq 398.8) \\
\text { Q3 }(>399.0, \leq 520.5) \\
\text { Q4 }(\geq 521.0) \\
P \text { for trend }\end{array}$ & $\begin{array}{c}\text { Reference } \\
-0.4736(-0.5890,-0.3583) \\
-0.7957(-0.9110,-0.6805) \\
-0.9918(-1.1086,-0.8750) \\
<0.001\end{array}$ & $\begin{array}{c}\text { Reference } \\
0.0817(0.0168,0.1466) \\
0.1310(0.0644,0.1977) \\
0.2418(0.1722,0.3115) \\
<0.001\end{array}$ & $\begin{array}{c}\text { Reference } \\
0.0891(0.0252,0.1530) \\
0.1628(0.0963,0.2293) \\
0.3006(0.2270,0.3743) \\
<0.001\end{array}$ \\
\hline $\begin{array}{l}\text { Stratified by age } \\
20-39 \text { years } \\
40-59 \text { years } \\
\end{array}$ & $\begin{array}{l}-0.0022(-0.0026,-0.0019) \\
-0.0019(-0.0023,-0.0016) \\
\end{array}$ & $\begin{array}{l}0.0006(0.0004,0.0008)^{* * *} \\
0.0004(0.0002,0.0006)^{* * *}\end{array}$ & $\begin{array}{l}0.0007(0.0005,0.0009)^{* * *} \\
0.0006(0.0004,0.0009)^{* * *}\end{array}$ \\
\hline $\begin{array}{l}\text { Stratified by race } \\
\text { Non-Hispanic white } \\
\text { Non-Hispanic black } \\
\text { Mexican American } \\
\text { Other race/ethnicity }\end{array}$ & $\begin{array}{l}-0.0020(-0.0023,-0.0016)^{* * *} \\
-0.0027(-0.0032,-0.0022)^{* * *} \\
-0.0023(-0.0028,-0.0017)^{* * *} \\
-0.0023(-0.0027,-0.0018)^{* * *}\end{array}$ & $\begin{array}{c}0.0007(0.0004,0.0009)^{* * *} \\
0.0005(0.0002,0.0007)^{* *} \\
0.0002(-0.0001,0.0005) \\
0.0001(-0.0001,0.0004)\end{array}$ & $\begin{array}{c}0.0009(0.0007,0.0012)^{* * *} \\
0.0004(0.0001,0.0007)^{*} \\
0.0003(-0.0000,0.0007) \\
0.0001(-0.0002,0.0003)\end{array}$ \\
\hline
\end{tabular}

Model 1: age and race were adjusted. Model 2: age, race, and body mass index were adjusted. Model 3: age, race, body mass index, education level, income-topoverty ratio, smoking status, drinking behavior, moderate activities, calcium supplement use, blood urea nitrogen, serum uric acid, total protein, serum estradiol level, serum phosphorus, and serum calcium were adjusted. In the subgroup analysis stratified by race, the model is not adjusted for the stratification variable itself. ${ }^{*} P<0.05,{ }^{* *} P<0.01$, and ${ }^{* * *} P<0.001$.

$(\beta=-0.0003,95 \% \mathrm{CI}:-0.0005$ to -0.0001$)$ groups and in the non-Hispanic white $(\beta=-0.0006,95 \% \mathrm{CI}:-0.0008$ to -0.0003 ) group in the fully adjusted model.

Results of the smooth curve fitting and generalized additive models to characterize the nonlinear associations between serum testosterone levels and lumbar BMD, ALMI, and AFMI are shown in Figures 1-3.

\section{Discussion}

Our population-based study revealed an association between serum testosterone levels and multiple indicators of body composition in men 20-59 years of age, namely a positive association with lumbar BMD and ALMI and a negative association with AFMI.

Osteoporosis in men is a growing public health concern, which remains underdiagnosed and underappreciated [14]. Sex hormones play an important role not only in the acquisition of bone mass but also in the maintenance of bone mass [15]. In a study of 60 schoolboys, the serum testosterone level was a major determinant of BMD at different pubertal stages [16]. In another study, a positive association between serum testosterone levels at the age of 12 years and a subsequent 6-year increase in BMD was identified [17]. A recent randomized clinical trial of men $\geq 65$ years of age reported that increasing the testosterone concentration 
TABle 4: Association between serum testosterone levels $(\mathrm{ng} / \mathrm{dL})$ and appendicular fat mass index $\left(\mathrm{kg} / \mathrm{m}^{2}\right)$.

\begin{tabular}{|c|c|c|c|}
\hline & $\begin{array}{c}\text { Model 1 } \\
\beta(95 \% \text { CI })\end{array}$ & $\begin{array}{c}\text { Model } 2 \\
\beta(95 \% \text { CI })\end{array}$ & $\begin{array}{c}\text { Model } 3 \\
\beta(95 \% \text { CI })\end{array}$ \\
\hline Serum testosterone levels (ng/dL) & $-0.0032(-0.0034,-0.0029)^{* * *}$ & $-0.0003(-0.0004,-0.0001)^{* * *}$ & $-0.0003(-0.0005,-0.0002)^{* * *}$ \\
\hline $\begin{array}{l}\text { Serum testosterone levels (quartile) } \\
\text { Q1 }(\leq 300.0) \\
\text { Q2 }(>300, \leq 398.8) \\
\text { Q3 }(>399.0, \leq 520.5) \\
\text { Q4 }(\geq 521.0) \\
P \text { for trend } \\
\end{array}$ & $\begin{array}{c}\text { Reference } \\
-0.6786(-0.7988,-0.5584) \\
-1.0777(-1.1978,-0.9576) \\
-1.4748(-1.5965,-1.3531) \\
<0.001 \\
\end{array}$ & $\begin{array}{c}\text { Reference } \\
-0.0714(-0.1308,-0.0120) \\
-0.0643(-0.1253,-0.0033) \\
-0.1259(-0.1896,-0.0622) \\
<0.001\end{array}$ & $\begin{array}{c}\text { Reference } \\
-0.0745(-0.1337,-0.0153) \\
-0.0775(-0.1391,-0.0158) \\
-0.1482(-0.2165,-0.0799) \\
0.001\end{array}$ \\
\hline $\begin{array}{l}\text { Stratified by age } \\
20-39 \text { years } \\
40-59 \text { years }\end{array}$ & $\begin{array}{l}-0.0036(-0.0040,-0.0033)^{* * *} \\
-0.0026(-0.0029,-0.0022)^{* * *}\end{array}$ & $\begin{array}{l}-0.0003(-0.0005,-0.0001)^{* * *} \\
-0.0002(-0.0004,-0.0001)^{*}\end{array}$ & $\begin{array}{c}-0.0004(-0.0006,-0.0002)^{* * *} \\
-0.0003(-0.0005,-0.0001)^{* *}\end{array}$ \\
\hline $\begin{array}{l}\text { Stratified by race } \\
\text { Non-Hispanic white } \\
\text { Non-Hispanic black } \\
\text { Mexican American } \\
\text { Other race/ethnicity }\end{array}$ & $\begin{array}{l}-0.0033(-0.0037,-0.0029)^{* * *} \\
-0.0036(-0.0041,-0.0030)^{* * *} \\
-0.0029(-0.0035,-0.0022)^{* * *} \\
-0.0024(-0.0028,-0.0020)^{* * *}\end{array}$ & $\begin{array}{c}-0.0004(-0.0007,-0.0002)^{* * *} \\
-0.0003(-0.0006,-0.0000)^{*} \\
0.0001(-0.0003,0.0004) \\
0.0001(-0.0001,0.0003)\end{array}$ & $\begin{array}{c}-0.0006(-0.0008,-0.0003)^{* * *} \\
-0.0002(-0.0005,0.0000) \\
0.0001(-0.0003,0.0005) \\
0.0002(-0.0000,0.0004)\end{array}$ \\
\hline
\end{tabular}

Model 1: age and race were adjusted. Model 2: age, race, and body mass index were adjusted. Model 3: age, race, body mass index, education level, income-topoverty ratio, smoking status, drinking behavior, moderate activities, calcium supplement use, blood urea nitrogen, serum uric acid, total protein, serum estradiol level, serum phosphorus, and serum calcium were adjusted. In the subgroup analysis stratified by race, the model is not adjusted for the stratification variable itself. ${ }^{*} P<0.05,{ }^{* *} P<0.01$, and ${ }^{* * *} P<0.001$

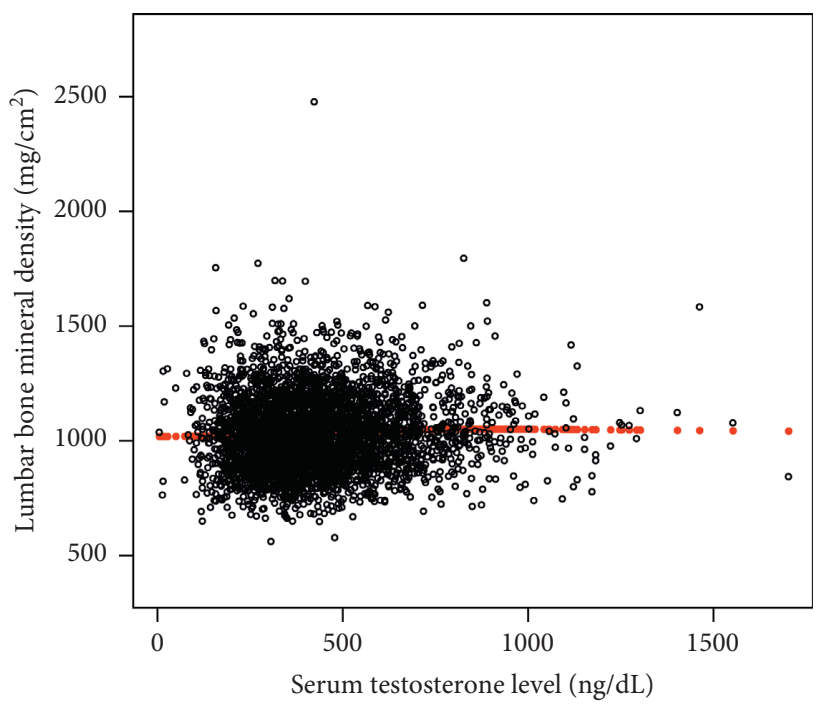

(a)

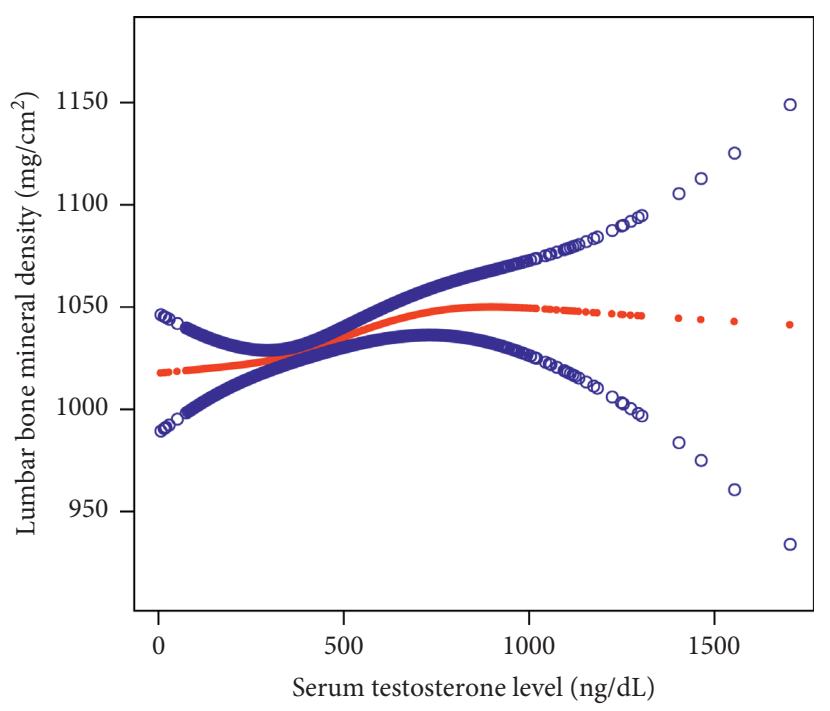

(b)

FiguRE 1: The association between serum testosterone levels and lumbar bone mineral density. (a) Each black point represents a sample. (b) Solid red line represents the smooth curve fit between variables. Blue bands represent the 95\% confidence interval from the fit. Age, race, body mass index, education level, income-to-poverty ratio, smoking status, drinking behavior, moderate activities, calcium supplement use, blood urea nitrogen, serum uric acid, total protein, serum estradiol level, serum phosphorus, and serum calcium were adjusted.

among older men with low testosterone levels has a positive effect on BMD and estimated bone strength [18]. One study specifically among men $<50$ years of age revealed that men with testosterone deficiency had greater odds of osteopenia and osteoporosis, with increases in BMD with testosterone treatment [19]. However, a recent meta-analysis showed no significant difference in total testosterone levels between primary osteoporotic and nonosteoporotic males [20].

It is well known that bodybuilders and athletes abuse testosterone to develop extra muscle bulk and strength [21]. However, testosterone under normal physiological conditions also helps regulate muscle mass and strength [22]. One study investigating the effect of long-acting testosterone in patients with congestive cardiac failure reported an improvement in muscle strength [23]. The findings from a previous NHANES (1999-2000) study of men, aged 18-85 years, revealed that higher testosterone at physiologic levels was associated with higher body lean mass and lower body fat mass [24]. However, other studies did not identify an effect on muscle strength $[25,26]$. In our study, the serum testosterone level was negatively associated with ALMI in the BMI-unadjusted model. However, in the models adjusted for 


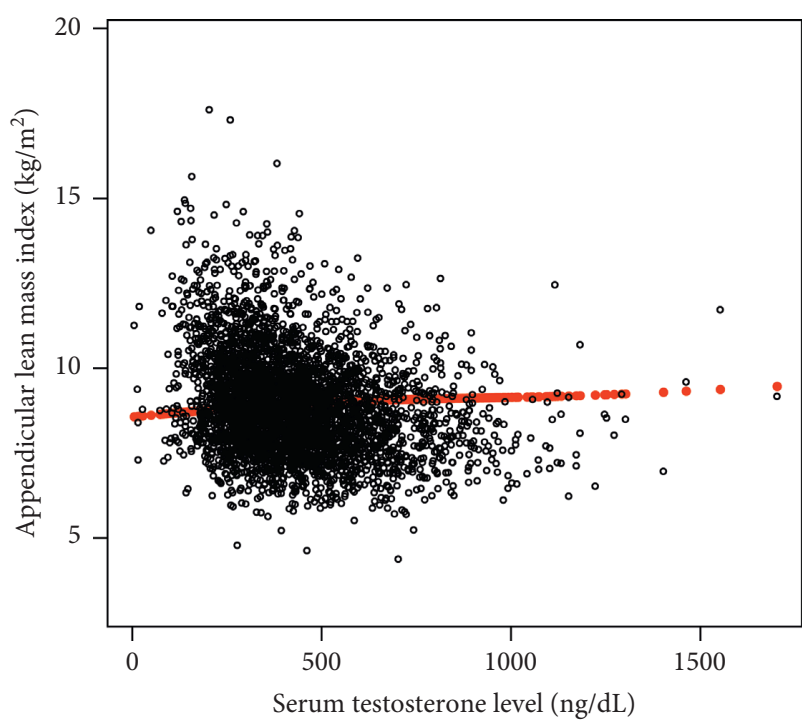

(a)

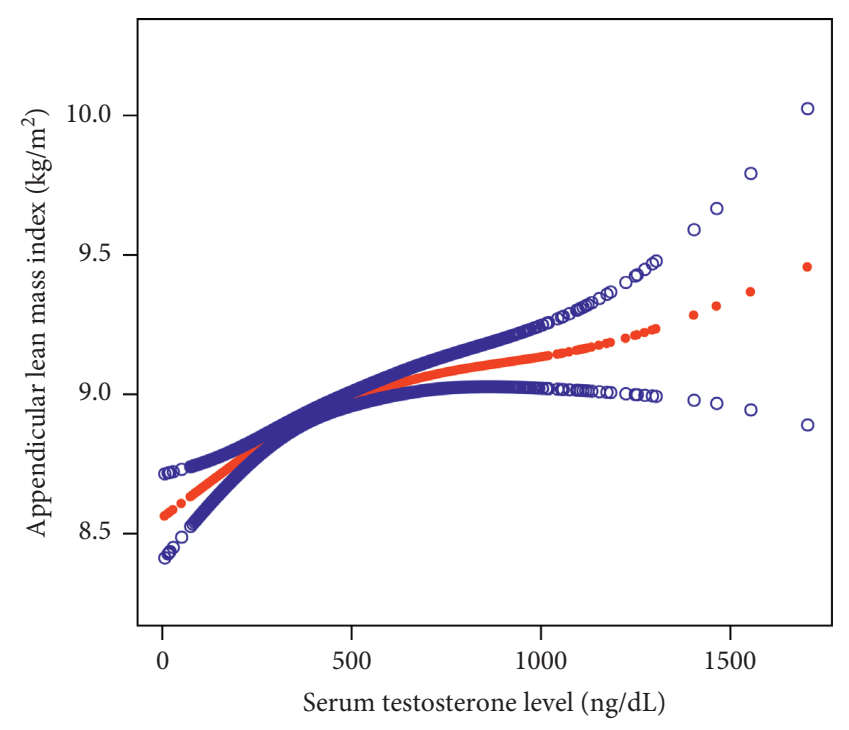

(b)

Figure 2: The association between serum testosterone levels and appendicular lean mass index. (a) Each black point represents a sample. (b) Solid red line represents the smooth curve fit between variables. Blue bands represent the $95 \%$ confidence interval from the fit. Age, race, body mass index, education level, income-to-poverty ratio, smoking status, drinking behavior, moderate activities, calcium supplement use, blood urea nitrogen, serum uric acid, total protein, serum estradiol level, serum phosphorus, and serum calcium were adjusted.

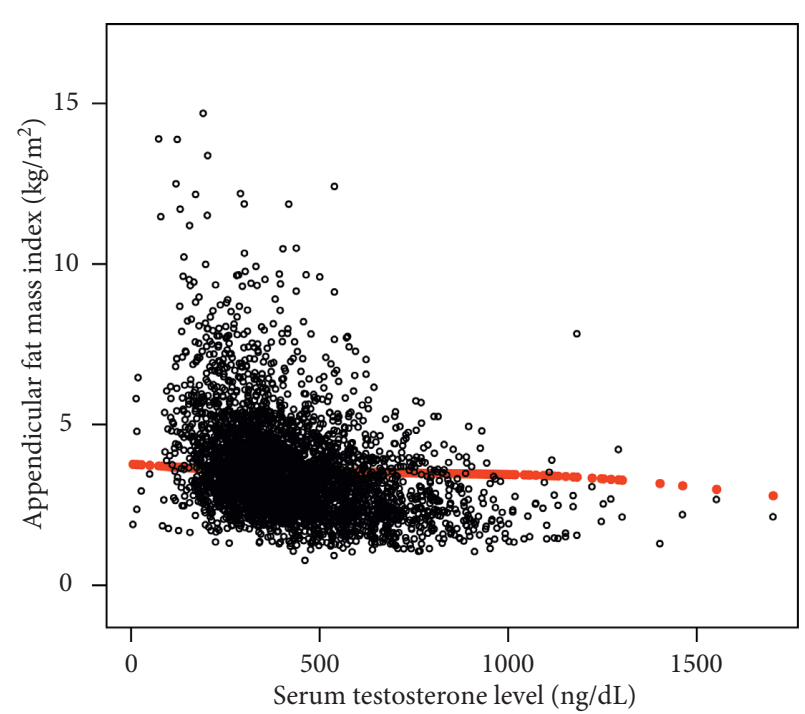

(a)

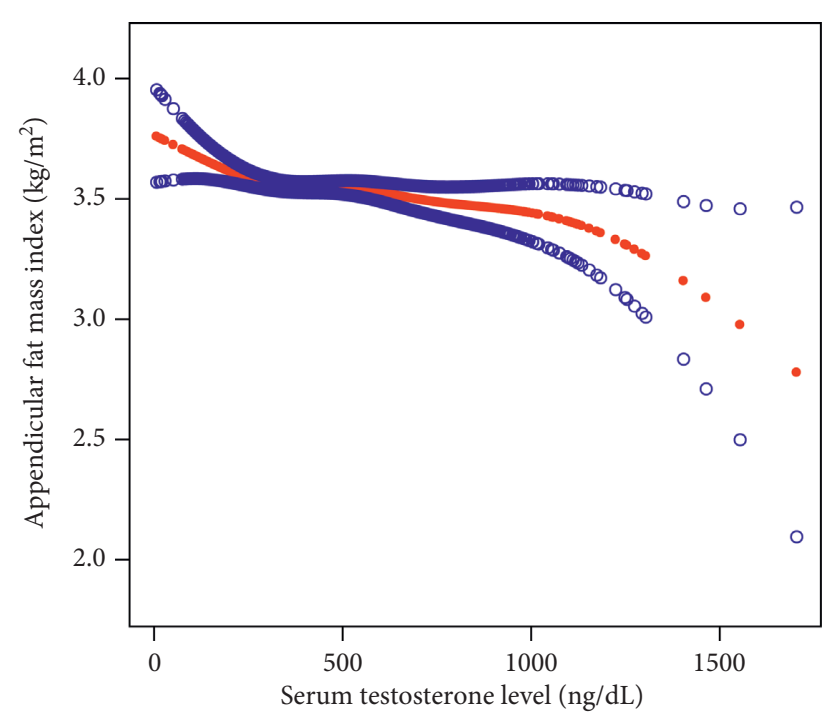

(b)

Figure 3: The association between serum testosterone levels and appendicular fat mass index. (a) Each black point represents a sample. (b) Solid red line represents the smooth curve fit between variables. Blue bands represent the $95 \%$ confidence interval from the fit. Age, race, body mass index, education level, income-to-poverty ratio, smoking status, drinking behavior, moderate activities, calcium supplement use, blood urea nitrogen, serum uric acid, total protein, serum estradiol level, serum phosphorus, and serum calcium were adjusted.

BMI, this association became positive. There are a variety of techniques used to estimate muscle quantity or mass, and multiple methods of adjusting the results for height or BMI. Our data showed that adjusting for height or BMI has a fundamental impact on the results. Therefore, these inconsistent findings related to the association between serum testosterone levels and BMD, and ALMI, may be attributed to heterogeneity among studies, including differences in study designs, participant selection, and control of confounding factors, especially BMI.

On the other hand, testosterone has been shown to exert an inhibitory effect on the incorporation of dietary fat into adipose tissue [27]. Testosterone also contributes to the maintenance of lower levels of fat mass by its conversion to estradiol [28]. A recent study of hypogonadal men reported an impressive effect of testosterone therapy on body weight, 
waist circumference, and BMI when compared with those not treated with testosterone [29].

The exact mechanism underpinning the association between testosterone and bone metabolism remains unclear. One possible explanation is that testosterone modulates bone remodeling either via aromatization of testosterone to estradiol or through direct activating of sex steroid receptors in bone cells [30]. Moreover, testosterone may have an indirect effect on bone mass through its anabolic effect of increasing muscle mass [31]. For individuals with low testosterone levels, adequate testosterone supplementation may be a useful adjunct therapy; however, the safety of testosterone intervention is of paramount concern as testosterone is a potent hormone acting on various organ systems.

To the best of our knowledge, our study is the largest report on the association between serum testosterone level and body composition in younger adult men $<60$ years of age. Our use of a large national representative sample increases the statistical strength for increased reliability of our results. Moreover, our age-stratified subgroup analysis allowed us to observe the association between the serum testosterone level and body composition in different life stages of youth and middle age. However, the limitations of our study need to be acknowledged. First, the cross-sectional design limits the inference of a causal correlation between the serum testosterone level and body composition. Second, because serum testosterone was not a static but a dynamic variable, measuring testosterone only once may cause some bias in testosterone levels used in the analysis. Third, in men's circulating plasma testosterone, approximately $2 \%$ to $3 \%$ is in free form, $33 \%$ to $54 \%$ is bound to albumin, and $44 \%$ to $65 \%$ is bound to sex hormone binding globulin, which loses its biological activity because of tight binding [32]. Bioavailable testosterone levels are not completely associated with testosterone levels. In our study, the association among free testosterone, bioactive testosterone or free androgen index, and body composition was not analyzed; this should be further clarified in future research.

In conclusion, we report a positive association between the serum testosterone level and lumbar BMD and ALMI, with a negative association to AFMI among men 20-59 years of age. Therefore, increasing testosterone levels may be beneficial to skeletal health in young and middle-aged men who have low testosterone levels. Further studies are needed to explore the threshold testosterone level that is beneficial for skeletal health without causing adverse events.

\section{Data Availability}

The data of this study are publicly available on the NHANES website.

\section{Ethical Approval}

The Ethics Review Board of the National Center for Health Statistics approved all NHANES protocols, and written informed consent was obtained from all participants.

\section{Conflicts of Interest}

The authors declare that they have no conflicts of interest.

\section{Authors' Contributions}

JJY, XJZ, and JXY contributed to data collection, analysis, and writing of the manuscript. ZXZ contributed to study design and writing of the manuscript.

\section{Acknowledgments}

The authors thank the staff and the participants of the NHANES study for their valuable contributions.

\section{References}

[1] M. A. Clynes, C. L. Gregson, O. Bruyère, C. Cooper, and E. M. Dennison, "Osteosarcopenia: where osteoporosis and sarcopenia collide," Rheumatology (Oxford), vol. 60, 2020.

[2] P. Wiedmer, T. Jung, J. P. Castro et al., "Sarcopenia-molecular mechanisms and open questions," Ageing Research Reviews, vol. 65, Article ID 101200, 2021.

[3] X. Li, J. Xu, B. Dai, X. Wang, Q. Guo, and L. Qin, “Targeting autophagy in osteoporosis: from pathophysiology to potential therapy," Ageing Research Reviews, vol. 62, Article ID 101098, 2020.

[4] B. Kirk, S. Phu, S. L. Brennan-Olsen, E. Bani Hassan, and G. Duque, "Associations between osteoporosis, the severity of sarcopenia and fragility fractures in community-dwelling older adults," European geriatric medicine, vol. 11, no. 3, pp. 443-450, 2020.

[5] V. Carnevale, E. Romagnoli, C. Cipriani et al., "Sex hormones and bone health in males," Archives of Biochemistry and Biophysics, vol. 503, no. 1, pp. 110-117, 2010.

[6] M. Velders and P. Diel, "How sex hormones promote skeletal muscle regeneration," Sports Medicine, vol. 43, no. 11, pp. 1089-1100, 2013.

[7] P. Dandona, S. Dhindsa, H. Ghanim, and F. Saad, "Mechanisms underlying the metabolic actions of testosterone in humans: a narrative review," Diabetes, Obesity and Metabolism, vol. 23, no. 1, pp. 18-28, 2021.

[8] G. Golds, D. Houdek, and T. Arnason, "Male hypogonadism and osteoporosis: the effects, clinical consequences, and treatment of testosterone deficiency in bone health," International journal of endocrinology, vol. 2017, Article ID 4602129, 15 pages, 2017.

[9] M. J. Shin, Y. K. Jeon, and I. J. Kim, "Testosterone and sarcopenia," The world journal of men's health, vol. 36, no. 3, pp. 192-198, 2018.

[10] M. Frost, T. L. Nielsen, K. Brixen, and M. Andersen, "Peak muscle mass in young men and sarcopenia in the ageing male," Osteoporosis International, vol. 26, no. 2, pp. 749-756, 2015.

[11] Z. Farrah and A. S. Jawad, "Optimising the management of osteoporosis," Clinical Medicine, vol. 20, no. 5, pp. e196-e201, 2020.

[12] Centers for disease control and prevention (CDC), national center for health statistics (NCHS), national health and nutrition examination survey laboratory protocol, sex steroid hormone-serum (TST_H). 2020, https://wwwn.cdc.gov/nchs/data/ nhanes/2013-2014/labmethods/TST_H_MET_Total_Estradiol_ and_Total_Testosterone.pdf. 
[13] C. L. Johnson, R. Paulose-Ram, C. L. Ogden et al., "National health and nutrition examination survey: analytic guidelines, 1999-2010," Data Evaluation and Methods Research, no. 161, pp. 1-24, 2013.

[14] R. A. Adler, "Update on osteoporosis in men," Best Practice \& Research Clinical Endocrinology \& Metabolism, vol. 32, no. 5, pp. 759-772, 2018.

[15] NIH Consensus Statement, "Osteoporosis prevention, diagnosis, and therapy," NIH Consensus Statement, vol. 17, no. 1, pp. 1-45, 2000.

[16] T. Pomerants, V. Tillmann, J. Jürimäe, and T. Jürimäe, “The influence of serum ghrelin, IGF axis and testosterone on bone mineral density in boys at different stages of sexual maturity," Journal of Bone and Mineral Metabolism, vol. 25, no. 3, pp. 193-197, 2007.

[17] R. Tamme, J. Jürimäe, E. Mäestu et al., "Association of serum testosterone at 12 years with a subsequent increase in bone mineral apparent density at 18 years: a longitudinal study of boys in puberty," Hormone research in paediatrics, vol. 91, no. 6, pp. 400-405, 2019.

[18] P. J. Snyder, D. L. Kopperdahl, A. J. Stephens-Shields et al., "Effect of testosterone treatment on volumetric bone density and strength in older men with low testosterone: a controlled clinical trial," JAMA internal medicine, vol. 177, no. 4, pp. 471-479, 2017.

[19] R. Kacker, W. Conners, J. Zade, and A. Morgentaler, "Bone mineral density and response to treatment in men younger than 50 years with testosterone deficiency and sexual dysfunction or infertility," The Journal of Urology, vol. 191, no. 4, pp. 1072-1076, 2014.

[20] Z. Y. Liu, Y. Yang, C. Y. Wen, and L. M. Rong, "Serum osteocalcin and testosterone concentrations in adult males with or without primary osteoporosis: a meta-analysis," BioMed Research International, vol. 2017, Article ID 9892048, 7 pages, 2017.

[21] H. G. Pope Jr., R. I. Wood, A. Rogol, F. Nyberg, L. Bowers, and S. Bhasin, "Adverse health consequences of performanceenhancing drugs: an Endocrine Society scientific statement," Endocrine Reviews, vol. 35, no. 3, pp. 341-375, 2014.

[22] K. Oki, T. D. Law, A. B. Loucks, and B. C. Clark, "The effects of testosterone and insulin-like growth factor 1 on motor system form and function," Experimental Gerontology, vol. 64, pp. 81-86, 2015.

[23] G. Caminiti, M. Volterrani, F. Iellamo et al., "Effect of longacting testosterone treatment on functional exercise capacity, skeletal muscle performance, insulin resistance, and baroreflex sensitivity in elderly patients with chronic heart failure," Journal of the American College of Cardiology, vol. 54, no. 10, pp. 919-927, 2009.

[24] J. G. Mouser, P. D. Loprinzi, and J. P. Loenneke, "The association between physiologic testosterone levels, lean mass, and fat mass in a nationally representative sample of men in the United States," Steroids, vol. 115, pp. 62-66, 2016.

[25] A. M. Kenny, A. Kleppinger, K. Annis et al., "Effects of transdermal testosterone on bone and muscle in older men with low bioavailable testosterone levels, low bone mass, and physical frailty," Journal of the American Geriatrics Society, vol. 58, no. 6, pp. 1134-1143, 2010.

[26] G. A. Wittert, I. M. Chapman, M. T. Haren, S. Mackintosh, P. Coates, and J. E. Morley, "Oral testosterone supplementation increases muscle and decreases fat mass in healthy elderly males with low-normal gonadal status," The journals of gerontology. Series A, Biological sciences and medical sciences, vol. 58, no. 7, pp. 618-625, 2003.
[27] P. Mårin, B. Odén, and P. Björntorp, “Assimilation and mobilization of triglycerides in subcutaneous abdominal and femoral adipose tissue in vivo in men: effects of androgens," Journal of Clinical Endocrinology \& Metabolism, vol. 80, no. 1, pp. 239-243, 1995.

[28] J. S. Finkelstein, H. Lee, S.-A. M. Burnett-Bowie et al., "Gonadal steroids and body composition, strength, and sexual function in men," New England Journal of Medicine, vol. 369, no. 11, pp. 1011-1022, 2013.

[29] F. Saad, G. Doros, K. S. Haider, and A. Haider, "Differential effects of 11 years of long-term injectable testosterone undecanoate therapy on anthropometric and metabolic parameters in hypogonadal men with normal weight, overweight and obesity in comparison with untreated controls: real-world data from a controlled registry study," International Journal of Obesity, vol. 44, no. 6, pp. 1264-1278, 2020.

[30] S. C. Manolagas, C. A. O’Brien, and M. Almeida, “The role of estrogen and androgen receptors in bone health and disease," Nature Reviews Endocrinology, vol. 9, no. 12, pp. 699-712, 2013.

[31] S. Bhasin, O. M. Calof, T. W. Storer et al., "Drug insight: testosterone and selective androgen receptor modulators as anabolic therapies for chronic illness and aging," Nature Clinical Practice Endocrinology \& Metabolism, vol. 2, no. 3, pp. 146-159, 2006.

[32] Z. Chen, X. Shen, K. Tian et al., "Bioavailable testosterone is associated with symptoms of depression in adult men," Journal of International Medical Research, vol. 48, no. 8, Article ID 300060520941715, 2020. 